\title{
Immuncheckpointhemmung
}

\section{Fatigue ist die häufigste Nebenwirkung}

US-Onkologen haben mehr als hundert klinische Studien für eine Bestandsaufnahme unerwarteter unerwünschter Wirkungen neuer Immuntherapeutika durchforstet. Fazit: Am häufigsten ist beim Einsatz der neuen Checkpointinhibitoren (Nivolumab, Pembrolizumab und Ipilimumab) mit Fatigue zu rechnen, und zwar bei bis zu jedem vierten Patienten. Durch die Kombination solcher Antikörper untereinander oder mit Chemotherapeutika, antiangiogenetisch wirksamen Präparaten oder zielgerichteten Therapien kann der Anteil der Betroffenen auf mehr als $70 \%$ steigen. Die Fatigue ist bei der Antikörpertherapie meist nur leicht ausgeprägt, kann aber mit einer Hypothyreose einhergehen.

Die häufigste unerwünschte Immunreaktion bei der Therapie mit Antikörpern gegen $\mathrm{PD}$ („,programmed cell death receptor)-1 und seinen Liganden 1 (u.a. PD-L1) ist Hautausschlag, der meist während des zweiten Therapiezyklus auftritt. Das kann ein makulopapulöses oder ein papulopustulöses Exanthem oder auch ein Sweet-Syndrom oder eine urtikarielle Dermatitis sein. Bei weniger als 5\% der Patienten mit dermatologischen Toxizitäten musste die Immuntherapie abgebrochen werden. Auch erhöhte Transaminasenwerte (AST, ALT) werden beobachtet: unter der Anti-CTLA-4-Therapie bei etwa $10 \%$ der Patienten und unter der Anti-PD1/PD-L1-Behandlung bei etwa $5 \%$. In Kombinationstherapien bei Patienten mit Leber- oder Nierenkrebs kann sich dieser Anteil auf $20 \%$ erhöhen.

Sowohl unter der Monotherapie mit Anti-PD1/PD-L1-Antikörpern, aber auch in Kombinationstherapien wurde über einige Fälle von Pneumonitis berichtet. Diese

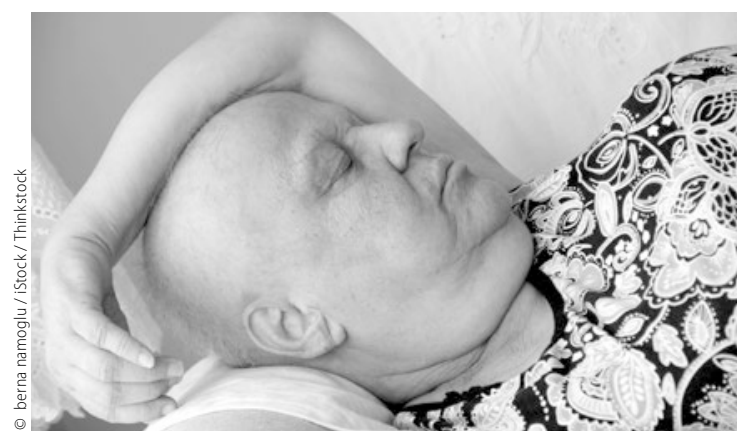

Fatigue tritt bei bis zu jedem vierten Patienten unter Checkpointinhibitoren auf.

unerwünschte Wirkung wurde unter einer Anti-CTLA-4-Therapie jedoch nicht beobachtet. Andererseits sei unter einer solchen Immunmonotherapie eher mit Durchfall oder Kolitis zu rechnen als bei einer Anti-PD1/PD-L1-Therapie (Grad3/4-Inzidenz: 5 vs. $3 \%$ ).

Peter Leiner

Naidoo J et al. Toxicities of the Anti-PD-1 and Anti-PD-L1 Immune Checkpoint Antibodies. Ann Oncol. 2015;26(12):2375-91.

\section{Hodgkin-Lymphom}

Die beiden wichtigsten Kombinationschemotherapie-Regimes zur Behandlung des Hodgkin-Lymphoms (HL) enthalten Bleomycin sowie ein Vincaalkaloid wie Vinblastin oder Vincristin. Um die zum Teil schweren Nebenwirkungen dieser Substanzen zu reduzieren, untersuchten Heinz Haverkamp und Kollegen, inwieweit sich die Dosis von Bleomycin und Vincaalkaloiden reduzieren lässt, ohne den Erfolg der Therapie zu riskieren.

Im Rahmen der Studien HD12 und HD15 der German Hodgkin Study Group (GHSG) haben sie das BEACOPPRegime abgewandelt. Bei 17,6\% der 3.309 analysierten HL-Patienten wurde die Behandlung mit Bleomycin unterbrochen und bei 32,6\% die Behandlung mit Vincristin.

Nach einem mittleren Follow-up von 59 bzw. 67 Monaten für das progressionsfreie Überleben (PFS) bzw. das Gesamtüberleben (OS) zeigten sich keine signifikanten Unterschiede zwischen den Patienten, die $\leq$ oder $>$ vier Zyklen Bleomycin erhielten. Beim 5-Jahres-PFS zeigte sich eine Differenz von 1,7 Prozentpunkten und beim 5-Jahres-OS von 1,5 Prozentpunkten. Auch bei Patienten, die $\leq$ oder $>$ drei Zyklen Vincristin erhielten, zeigten sich keine signifikanten Unterschiede: Sie lagen bei -1,3 Prozentpunkten für das 5-Jahres-PFS und bei -0,1 Prozentpunkten für das 5-Jahres-OS.

Eine Unterbrechung von Bleomycin oder Vincristin aufgrund medikamentenspezifischer Nebenwirkungen beeinflusst die Wirksamkeit der Therapie demnach also nicht. Die Gründe dafür sind bislang unklar. Es ist möglich, dass Bleomycin und Vincristin bei jenen Patienten, die besonders unter Nebenwirkungen durch diese Chemotherapeutika leiden, auch besonders wirksam sind und deshalb weniger Zyklen ausreichen; möglicherweise haben Bleomycin und Vincristin aber auch gar keine so große Bedeutung für den Erfolg der Therapie. Dies sollte in weiteren Studien untersucht werden. Christina Berndt

\footnotetext{
Haverkamp H et al. Impact of Bleomycin and Vincristine Dose Reductions in Patients With Advanced Hodgkin Lymphoma Treated With BEACOPP: An Analysis of the German Hodgkin Study Group HD12 and HD15 Trials. J Clin Oncol. 2015;33(22):2430-6.
}

\section{kurz notiert}

\section{Inhibition der Fettsäuresynthase bei Ovarialkarzinom}

Wiener Forscher versuchen die Therapie des Ovarialkarzinoms zu verbessern, indem sie zwei grundlegende Zellregulationswege gleichzeitig inhibieren. Einer dieser Wege betrifft die onkogene Fettsäuresynthase, die bei $80 \%$ der Ovarialkarzinome überexprimiert ist. Die Hemmung der Fettsäuresynthase löst eine komplexe Stressantwort aus, die in einen durch die PI3-Kinase und den mTOR-Komplex 1 vermittelten Signalweg eingreift. In-vitro-Versuche zeigten, dass die Inhibition dieses Signalwegs entscheidend für die Antitumoraktivität von Fettsäuresynthesehemmern ist [Wagner Ret al. ECC. 2015;Abstr 269]. Als nächster Schritt ist vorgesehen, Inhibitoren von Signalwegen der Zellteilung mit Fettsäuresynthesehemmern zu kombinieren. 